\title{
Ensino sobre gerenciamento de resíduos na graduação em enfermagem: realidade, reflexões e propostas
}

Teaching about waste management in undergraduate nursing: reality, reflections and proposals

Enseñanza sobre la gestión de residuos en enfermería de pregrado: realidad, reflexiones y propuestas

Paulyne Souza Silva Guimarães ${ }^{1 *}$, Rosana Quintella Brandão Vilela ${ }^{1}$, Silvana Maria Barros de Oliveira' ${ }^{1}$, Paulo Jorge Torres Guimarães Silva², Rosane Pereira dos Reis ${ }^{3}$, Esvaldo dos Santos Silva $^{3}$, Alexandre de Souza Lima², Renné Costa da Silva².

\section{RESUMO}

Objetivo: Conhecer a percepção dos discentes de Enfermagem sobre Gerenciamento de Resíduos de Serviços de Saúde (GRSS). Métodos: Estudo de abordagem qualitativa descritiva, desenvolvido em uma universidade pública do nordeste brasileiro. Utilizou-se da técnica de Grupo Focal (GF) com sete discentes do quarto período do curso de graduação em Enfermagem. Resultados: No contexto teórico, o ensino sobre GRSS mostrou-se fragmentado, carecendo de aprofundamento e amplitude conceitual. No contexto prático, constatou-se que as experiências vivenciadas acontecem, na maioria das vezes, no ambiente hospitalar, requerendo cenários de práticas diversificados. Conclusão: $O$ estudo constatou que existem fragilidades no processo de formação dos enfermeiros frente à temática GRSS. Possivelmente, esse fato está associado à fase do curso vivenciada pelos discentes pesquisados. Ainda assim, cabe à academia fomentar e estimular o aprendizado sobre o tema por meio de metodologias de ensino inovadoras, contextualizadas, articuladas e problematizadas, que sejam capazes de instrumentalizar os discentes para o enfrentamento dos Resíduos de Serviços de Saúde (RSS) de forma responsável e consciente.

Palavras-chave: Resíduos de serviços de saúde, Educação em enfermagem, Competência profissional, Gerenciamento de resíduos.

\section{ABSTRACT}

Objective: To know the perception of nursing students about Health Services Waste Management (GRSS). Methods: Study of a descriptive qualitative approach, developed at a public university in northeastern Brazil. The Focus Group ( $F G$ ) technique was used with seven students from the fourth period of the undergraduate nursing course. Results: In the theoretical context, teaching on GRSS was fragmented, lacking in depth and conceptual breadth. In the practical context, it was found that the experiences they take place, most of the time, in the hospital environment, requiring diverse practice scenarios. Conclusion: The study found that there are weaknesses in the process of training nurses regarding the GRSS theme. This fact is possibly associated with the course phase experienced by the researched students. Even so, it is up to the academy to foster and stimulate learning on the topic through innovative, contextualized, articulated and problematized teaching methodologies, which are capable of instructing students to cope with Health Services Residues (RSS) in a responsible manner. and conscious.

Keywords: Waste health services, Nursing education, Professional competence, Waste management.

1 Universidade Federal de Alagoas (UFAL), Maceió - AL. *E-mail: paulyne.guima@gmail.com

2 Universidade de Brasília (UnB), Brasília - DF.

${ }^{3}$ Faculdade Estácio de Alagoas, Maceió - AL. 


\section{RESUMEN}

Objetivo: Conocer la percepción de los estudiantes de enfermería sobre la Gestión de Residuos de Servicios de Salud (GRSS). Métodos: Estudio de enfoque descriptivo cualitativo, desarrollado en una universidad pública del noreste de Brasil. Se utilizó la técnica Focus Group (FG) con siete estudiantes del cuarto período de la carrera de enfermería. Resultados: En el contexto teórico, la enseñanza sobre GRSS estaba fragmentada, carecía de profundidad y amplitud conceptual. En el contexto práctico, se encontró que las experiencias se desarrollan, la mayoría de las veces, en el ámbito hospitalario, requiriendo de diversos escenarios de práctica. Conclusión: El estudio encontró que existen debilidades en el proceso de formación de enfermeras en el tema GRSS. Este hecho posiblemente esté asociado a la fase del curso vivida por los estudiantes investigados. Aun así, le corresponde a la academia fomentar y estimular el aprendizaje sobre el tema a través de metodologías didácticas innovadoras, contextualizadas, articuladas y problematizadas, que sean capaces de instruir a los estudiantes para el afrontamiento de los Residuos de Servicios de Salud (RSS) de manera responsable y consciente.

Palabras clave: Servicios de salud de residuos, Educación de enfermería, Competencia profesional, Gestión de residuos.

\section{INTRODUÇÃO}

A sociedade enfrenta sérios desafios, entre os quais, a complexidade e a diversidade existentes nos Resíduos Sólidos Urbanos (RSU). Entre os diversos tipos de RSU que resultam das ações antrópicas, os Resíduos de Serviços de Saúde (RRS), dentro de uma dimensão maior, constituem um desafio com interfaces, uma vez que além da questão ambiental inerente a qualquer resíduo, apresentam risco para a saúde do trabalhador e para a saúde pública (ZARPELÃO RZN, et al., 2016).

Moreira AMM e Gunther W (2016) afirmam que os RSS representam um sério problema de saúde pública para a sociedade e para o ambiente em razão da presença de organismos patogênicos ou de suas toxinas, produtos químicos de natureza diversa, a saber: fármacos, quimioterápicos, fixadores de RX, reagentes, saneantes, bem como material contendo rejeitos radiológicos. Nesse aspecto, há uma preocupação mundial quanto ao descarte inadequado ou aleatório dos resíduos, visto que pode gerar agravos à saúde dos trabalhadores que os coletam, como também acidentes fatais semelhantes ao rompimento da cápsula de Cesio-137.

O Gerenciamento dos Resíduos de Serviços de Saúde (GRSS) adequado e seguro, de acordo com a Agência Nacional de Vigilância Sanitária (ANVISA), é um processo articulado e contínuo que depende de atualizações científicas e técnicas, regramento por instrumentos leais e normativos, além da qualificação dos recursos humanos (BRASIL, 2018). Cabe destacar que as Diretrizes Curriculares Nacionais da Enfermagem (DCN/ENF) orientam o perfil do formando egresso com formação generalista capaz de atuar nos problemas/situações de saúde-doença, com senso de responsabilidade social e compromisso com a cidadania, como promotor integral da saúde do ser humano (BRASIL, 2001). O referido documento, além de outros pressupostos, descreve que o enfermeiro deve estar apto a fazer o gerenciamento e a administração tanto da força de trabalho quanto de recursos físicos, materiais e de informação.

Moreschi C, et al. (2014) e Sanchez APM, et al. (2018) advertem que o GRSS nem sempre é incorporado nos conteúdos teóricos e nas atividades práticas das Instituições de Ensino Superior (IES), podendo este profissional não reservar tempo para aprender a gerenciar os Resíduos de Serviços de Saúde (RSS), o que dificulta a consolidação de novos conceitos e proatividade diante da problemática.

Nesse contexto, as IES, como geradoras e difusoras do conhecimento, precisam assumir seu papel perante a temática dos resíduos de serviços de saúde (OLIVEIRA ACR, et al., 2019). A necessidade de maiores contribuições para a questão bem como o interesse como preceptora em contribuir com 0 aprimoramento do curso pesquisado, justificaram o desenvolvimento deste estudo, que teve como pergunta de investigação: Na perspectiva dos discentes, como o tema Gerenciamento de Resíduos de Serviços de Saúde vem sendo abordado na graduação? 
$\mathrm{Na}$ intenção de responder a essa questão, o estudo teve como objetivo conhecer a percepção dos discentes de Enfermagem sobre os Resíduos de Serviços de Saúde em um curso de uma universidade pública federal do nordeste brasileiro.

\section{MÉTODOS}

Foi realizada uma pesquisa de campo descritiva e analítica com abordagem qualitativa. Os sujeitos da pesquisa foram os discentes do curso de Enfermagem regulamente matriculados e que cursaram o $3^{\circ}$ período. Foram excluídos do estudo os discentes que estavam afastados por qualquer motivo no período da coleta de dados.

Para alcançar o objetivo desta pesquisa, utilizou-se a técnica de Grupo Focal (GF). Em se tratando de GF, destaca-se que a abordagem por meio dessa técnica propõe a construção de conhecimento em espaços de intersubjetividade, em que muitas vozes focadas em tópicos específicos e diretivos trocam experiências, conceitos e opiniões (SOARES MI, et al., 2016).

O encontro foi realizado no dia 10 de abril de 2019, em uma sala reservada, localizada na instituição pesquisada. O cenário de investigação refere-se ao curso de graduação em Enfermagem de uma universidade pública, localizada no nordeste brasileiro, sendo aprovado pelo Comitê de Ética em Pesquisa (CEP) da Universidade Federal de Alagoas (UFAL), conforme Parecer ํㅜ 3.099.358.

$\mathrm{Na}$ busca dos discentes de Enfermagem, inicialmente, foi efetuado contato com os representantes de turma dos $4^{\circ}, 5^{\circ}, 6^{\circ}, 7^{\circ}$ e $8^{\circ}$ períodos do curso estudado. Em seguida, foi enviado um convite por e-mail para as respectivas turmas, além da divulgação do convite da pesquisa no mural do respectivo curso. Neste recorte do estudo, participaram sete discentes do $4^{\circ}$ período, sendo: cinco mulheres e dois homens. A faixa etária foi de 19 a 21 anos, e nenhum dos participantes havia realizado qualquer atividade complementar que abordasse a temática dos resíduos. Tal recorte se justifica, em razão dos achados encontrados e por recomendação da banca de qualificação.

Destaca-se que os princípios de biossegurança são abordados a partir do $2^{0}$ ano do curso e, especificamente, a temática em estudo no 3 o período. O tamanho da amostra atendeu às recomendações de Minayo MCS (2015). A referida autora sugere que, para a obtenção de resultados confiáveis na pesquisa qualitativa, o número de participantes deve atender à extensão do objeto e à complexidade do estudo.

Após informar a todos sobre a gravação das discussões com o auxílio de dois dispositivos de telefone móvel (smartphone), reforçou-se a necessidade de respeito e o anonimato entre eles. Foram fornecidos esclarecimentos referentes ao Termo de Consentimento Livre e Esclarecido (TCLE). O documento foi lido e entregue a cada participante para ciência e assinatura.

Para nortear o GF, foram utilizadas as seguintes questões: O que você entende por RSS? Como você percebe a importância do enfermeiro frente ao GRSS? Cite oportunidades de aprendizagem sobre GRSS durante o curso.

A transcrição do áudio foi realizada na íntegra e os participantes foram identificados com a letra "D" de discentes seguido de um numeral conforme a sequência inicial de participação no grupo. O cenário de práticas e as disciplinas citadas nas discussões foram identificados com as letras do alfabeto para evitar qualquer possibilidade de identificação. Os dados produzidos com base no GF foram armazenados, transcritos, sistematizados, categorizados e analisados.

\section{RESULTADOS E DISCUSSÃO}

Partindo destes pressupostos e baseado nas impressões pessoais, valores e opiniões, explicitados nas entrevistas, o processo de análise possibilitou a identificação de categorias temáticas empíricas e suas subcategorias.

\section{Os saberes dos discentes sobre RSS}

Essa categoria é oriunda da identificação de três subcategorias: Conceitos atribuídos aos RSS e GRSS; Classificação e etapas do manejo do RSS; e Consequências do descarte inadequado dos RSS. 
A Agência Nacional de Vigilância Sanitária (ANVISA), por meio da Resolução da Diretoria Colegiada RDC nำ222/2018 (BRASIL, 2018), define como estabelecimentos geradores de RSS as instituições sanitárias que prestam cuidado à saúde humana e animal. Nas falas, os discentes concebem os RSS como toda sobra de produto proveniente da assistência à saúde, gerados nos hospitais e unidades de saúde. Nesse contexto, verifica-se a necessidade dos discentes em ampliar o olhar quanto aos locais de geração dos RSS, uma vez que não se limitam apenas as instituições hospitalares e unidades de saúde.

"São produtos gerados após assistência à saúde" (D4).

"[...] é todo lixo gerado nos hospitais e nas unidades de saúde" (D7).

A literatura demonstra que os estabelecimentos de Atenção Primária à Saúde (APS) secundária e terciária, durante o processo de cuidar, geram resíduos e efluentes que necessitam de um gerenciamento adequado (ZAJAC MAL, et al., 2016). Outro conceito explorado durante o GF foi o GRSS. Esse gerenciamento consiste em uma estratégia de gestão pautada nos aspectos técnico-operacionais legais e normativos objetivando o controle e a diminuição dos riscos e minimização dos resíduos, com vistas à proteção sanitária e ambiental (BRASIL, 2018). Para os discentes envolvidos no estudo, o GRSS esteve muito atrelado à biossegurança:

"Acho que tem a ver com a segurança a nós, tipo não só de enfermeiros, técnicos, mais de todos dentro do hospital [...] então o uso dos EPIs" (D6).

"[...] o lixo pode levar a infecção cruzada quando o profissional não lava as mãos ou troca a luva depois do procedimento" (D7).

A biossegurança compreende um conjunto de normas e decisões técnicas que visam, prioritariamente, à prevenção e minimização de riscos, propondo mudanças no cotidiano dos profissionais, bem como adequações nos serviços de saúde (BORGES GG, et al., 2014). Entre as medidas de biossegurança, destacase o uso dos Equipamentos de Proteção Individual (EPIs) que se destinam a proteger o enfermeiro e a equipe de enfermagem nas ações técnicas que envolvam riscos de exposição ou quando houver manuseio de artigos e materiais biológicos, produtos, químicos ou radioativos.

As resoluções da ANVISA e do Conselho Nacional do Meio Ambiente (CONAMA) classificam os RSS em cinco grupos, de acordo com as suas características, a saber: Grupo A - infectantes (subdivididos em: A1, A2, A3, A4 e A5); Grupo B - químicos; Grupo C - rejeitos radioativos; Grupo D - comuns; Grupo E perfurocortantes (SILVA DP, et al., 2019). Nesse estudo, observou-se nos relatos dos discentes uma diversidade de RSS, com destaque para os resíduos biológicos, comuns e perfurocortantes. Não foram mencionados os resíduos químicos e radioativos.

"[...] material que a gente usa tipo: gaze, seringa, luva, agulha" (D1).

"[...] máscaras, os resíduos orgânicos, papel tolha” (D2).

"Fluídos dos pacientes, resíduos da assistência, os reutilizáveis de embalagens, copos" (D7).

Um estudo envolvendo docentes, discentes e egressos da área da Saúde concluiu que os discentes pesquisados atribuíram maior significância aos resíduos contaminados em razão de que podem fazer mal à saúde, ao passo que os docentes e egressos afirmaram que os resíduos vão além dos infectantes (MORESCHI C, et al., 2014).

Durante o GF, os discentes mencionaram as etapas de segregação, armazenamento e tratamento:

"[...] deve ser a forma como é tratado o material contaminado, perfurocortante e material cirúrgico, aquele que vem da cirurgia" (D5).

"[...] é o armazenamento desses resíduos num lugar apropriado para que depois ele sofra a incineração" (D3).

"A gente aprendeu os processos que se tinham depois que o lixo sai do hospital até chegar na incineração [...] tipo a separação, pesagem quem fazia cada etapa" (D7). 
Um estudo avaliou o conhecimento e a atitude dos alunos do curso de graduação em Odontologia, sobre o descarte e o acondicionamento dos resíduos dos materiais mais utilizados na rotina odontológica, evidenciando o desconhecimento sobre a forma correta do descarte e acondicionamento dos resíduos de saúde por grande parte dos participantes (GARBIN AJI, et al., 2015). Essa pesquisa mostrou que os participantes detêm um conhecimento parcial sobre a classificação e as diferentes etapas do manejo dos RSS. Essa questão é preocupante, porque a identificação e a compreensão do processo de manejo em sua completude, desde a geração até a disposição final, são essenciais para os enfermeiros em formação. Somente por meio desse conhecimento será possível manusear os RSS de forma consciente e comprometida.

Os discentes, de forma geral, conseguiram descrever as consequências dos RSS quando descartados de forma inadequada, no aspecto relacionado tanto ao risco biológico, quanto socioambiental e econômico.

"Se descartado errado os resíduos podem contribuir para transmissão de doenças como o vírus do HIV e da Hepatite B para os trabalhadores da saúde" (D5).

"Risco de contaminação para o paciente e para os profissionais de enfermagem e de outras áreas" (D4).

"Os perfurocortantes quando manipulados de forma errada podem aumentar os riscos de acidentes de trabalho" (D3).

No que tange a avaliação dos riscos potenciais dos RSS, identificam-se principalmente o biológico e o ambiental. Relativo ao risco biológico deve-se considerar a cadeia de transmissibilidade das doenças pelas vias respiratórias, digestiva e pela absorção cutânea e mucosa durante a atividade ocupacional, com ênfase nos materiais infectantes ou metais pesados. No tocante ao risco ambiental, é considerada a probabilidade da ocorrência de efeitos adversos ao meio ambiente e ocorre decorrente da ação de agentes físicos, químicos ou biológicos (BRASIL, 2006).

A questão ambiental tem se tornado um determinante do processo saúde- doença, caracterizado por problemas sanitários que afetam a população (PETRES AA e DA ROS MA, 2018).

A responsabilidade socioambiental no manejo dos RSS foi citada pelo participante:

"O lixo pode causar infecção hospitalar e contaminação do meio ambiente se desprezado incorretamente" (D7).

Em relação aos riscos ao meio ambiente, os RSS quando lançados a céu aberto ou sem o devido tratamento acabam contaminando o solo, a água e o ar. Além disso, também, proporciona sérios riscos aos catadores, principalmente por meio de lesões provocadas por materiais cortantes ou perfurocortante, ingestão de peças anatômicas humanas, alimentos contaminados ou intoxicação por material particulado contaminado em suspensão (SILVA DF, et.al., 2014).

Os riscos econômicos ocasionados pelo descarte inadequado de RSS foram citados pelos estudantes. Sobre este aspecto, os sujeitos se posicionaram:

"Posso dizer que a falta de gerenciamento para o hospital implica em gastos e para o paciente uma possível regressão na alta hospitalar" (D2).

"Acho que se não descartar da maneira certa aumenta o volume do contaminado e termina pagando mais" (D3).

Cafure VA e Graciolli-Patriarcha SR (2015), estudando os impactos dos RSS, identificaram um distanciamento entre teoria e prática dentro e fora dos serviços, seja por parte dos gestores, profissionais da Saúde e até mesmo pelos trabalhadores que manuseiam diariamente esses resíduos. Dessa maneira, podese depreender que o contato com a prática constitui-se um caminho diferenciado que permite ampliar os saberes dos discentes sobre os RSS. É importante a manutenção e a criação de novos espaços de reflexão sobre o tema, em consonância com as políticas e os pressupostos teóricos, promovendo debates e estratégias voltados à reformulação das práticas em saúde. 


\section{O fazer do enfermeiro frente à gestão de RSS, na percepção dos discentes}

Nesta categoria discute-se a concepção dos participantes da pesquisa sobre o fazer do profissional de enfermagem no desenvolvimento do PGRSS. As subcategorias que contribuíram com o aparecimento deste conjunto foram: GRSS: um ofício regulado tecnicamente, e Educação Permanente em Saúde (EPS): ferramenta para melhor agir no trabalho.

Há, na literatura, estudos que revelam maior envolvimento da enfermagem no manejo dos RSS, por assumirem papéis de gestão de serviços. Este fato tem levado outros profissionais a se sentirem desobrigados de tal função (CORRÊA LB, et al., 2007). Nesse estudo, percebeu-se que esse entendimento foi identificado pelo discente:

"Os enfermeiros são responsáveis pela gerência dos resíduos dentro da instituição e acredito que seja através de protocolos" (D4).

Cabe ao enfermeiro, como líder da equipe de enfermagem, atuar no PGRSS, pois é responsável pela maior parte da prestação da assistência ao cuidado, podendo, portanto, interferir significativamente no controle das infecções hospitalares e na priorização da gestão integrada dos RSS (COFEN, 2005; MARTINS DF e BENITO LAO, 2016; BENTO DG, et al., 2017; PEREIRA MS, et al., 2013). Os discentes, apesar de não lembrarem o parecer do COFEN que habilita o enfermeiro a assumir a responsabilidade técnica do PGRSS, reconheceram a necessidade desse conteúdo ser aprofundado no ensino da graduação.

"Os enfermeiros precisam incorporar na academia essa questão e botar em prática, buscar conhecimentos e treinar a equipe de enfermagem" (D2).

O PGRSS é um documento importante que auxilia a gestão em relação à proteção da saúde pública, do trabalhador e do meio ambiente. Ainda descreve as ações relativas ao gerenciamento de resíduos, considerando suas características e os riscos atribuidos a cada tipo de residuo, abrangendo as etapas de geração, identificação, segregação, acondicionamento, coleta, armazenamento, transporte, destinação e disposição final ambientalmente adequada (BRASIL, 2018). Os participantes do estudo não identificaram qualquer oportunidade de acesso ao PGRSS, seja na academia seja na rede de serviços de saúde (cenários de prática). Eles advertiram que o enfermeiro, consciente ou não, está exposto aos riscos no entorno da assistência.

\section{"É preciso que o enfermeiro acorde para os riscos que acaba sendo inserido quando é imprudente e negligente" (D6).}

Para tanto, o PGRSS pode ser considerado como uma etapa fundamental para que se realize uma completa promoção da prevenção da saúde pública, uma vez que, ao utilizá-lo e transformá-lo num modelo a ser seguido pela instituição, o enfermeiro estará atuando de forma definitiva no aspecto de prevenção de agravos à saúde (BATAGLIN MS, et al., 2012). Nesse estudo, identificou-se que, na percepção dos discentes do quarto período, existe um baixo protagonismo na teoria e prática do GRSS, por parte da IES, dentro e fora dos serviços de saúde. Possivelmente, esta visão está relacionada ao período do curso em que os participantes se encontram.

Para a Organização Pan-Americana de Saúde (OPAS) os termos Educação Permanente em Saúde (EPS) e educação continuada são diferentes, e considera a última mais reducionista. A equiparação conceitual se dá pelo fato de ambas implicarem a propagação de conhecimentos, porém a educação permanente possibilita espaços de discussão e de reflexão sobre a prática, o que pode resultar em melhor assistência prestada pelos profissionais da Saúde (BRASIL, 2018). Nessa pesquisa, os discentes perceberam a importância dessa estratégia para o fomento de mudança no comportamento do ambiente de trabalho:

"Na educação continuada o enfermeiro é o responsável por orientar e treinar a equipe sobre essa questão" (D3).

"No dia a dia é possível perceber a necessidade de treinamentos, manter as pessoas atualizadas sobre os resíduos da saúde, a gente só olha a parte técnica, mas não vê todo o resto" (D5). 
Vital MSB e Guimarães PSS (2018) afirmam ser necessários investimentos em processo de EPS, contribuindo para a consolidação de valores ambientais, promovendo qualidade de vida associada à preservação e à sustentabilidade. Estudos reconhecem a importância organizacional das fontes geradoras de RSS quanto ao manejo correto dos seus resíduos, e destacam a necessidade de despertar a consciência coletiva dos sujeitos acerca das responsabilidades individuais na atenção com os resíduos gerados (SZCZERBOWSKI AC e MORAIS CR, 2017; DOI KM e MOURA MSS, 2011). A EPS constitui-se como um elemento fundamental para o desenvolvimento da coletividade que vive em constantes transformações. Possibilita incorporar as novas tecnologias na tentativa de desencadear processos que assegurem a cidadania.

Os resultados obtidos permitiram observar que a abordagem do conteúdo referente ao GRSS foi informada pelos discentes investigados, no entanto, foi avaliada como insuficiente. Até o quarto período da formação acadêmica de Enfermagem, o tema é vivenciado a partir de uma disciplina teórica, porém de forma fragmentada, carecendo de aprofundamento e amplitude conceitual. Pode-se depreender que a diversificação de cenários de aprendizagem constitui-se uma estratégia para ampliar os saberes dos discentes sobre RSS.

\section{CONCLUSÃO}

O estudo constatou que existem fragilidades no processo de formação dos enfermeiros frente à temática gerenciamento de resíduos de serviços de saúde. Possivelmente, esse fato esteja associado à fase do curso vivenciada pelos discentes pesquisados. Ainda assim, cabe à academia deve fomentar e estimular o aprendizado sobre o tema por meio de metodologias de ensino inovadoras, contextualizadas, articuladas e problematizadas, que sejam capazes de instrumentalizar os discentes para o enfrentamento dos RSS de forma responsável e consciente. Destarte, esses resultados merecem ser investigados em estudos futuros, para aprofundamento, buscando soluções para melhorar o ensino sobre o tema.

\section{REFERÊNCIAS}

1. BATAGLIN MS, et al. Conhecimento da Equipe de Enfermagem sobre a Segregação dos Resíduos Sólidos em Ambiente Hospitalar. Ensino, Saúde e Ambiente, 2012; 5 (3): 68-83.

2. BENTO DG, et al. O gerenciamento de resíduos de serviço de saúde sob a ótica dos profissionais de enfermagem. Texto Contexto - Enfermagem, 2017; 26(1).

3. BORGES GG, et al. Biossegurança na Central de Quimioterapia: o Enfermeiro frente ao Risco Químico. Revista Brasileira de Cancerologia, 2014; 60 (3): 247-250.

4. BRASIL. Manual do Ministério da Educação. 2001. Disponível em: http://portal.mec.gov.br/index.php?option=com_docman\&view=download\&alias=44661-rces003-16pdf\&category_slug=junho-2016-pdf\&Itemid=30192. Acessado em: 23 jul. 2019.

5. BRASIL. Manual do Ministério da Saúde. $2006 . \quad$ Disponível em: https://www.anvisa.gov.br/servicosaude/manuais/manual_gerenciamento_residuos.pdf. Acessado em: 06 dez. 2020.

6. BRASIL. Manual do Ministério da Saúde. 2018. Disponível em: https://spdata.com.br/resolucao-rdc-no-222-de-28-demarco-de-2018/. Acessado em: 06 dez. 2020.

7. BRASIL. Resolução COFEN no 303. 2005. Disponível em: https://www.portaldaenfermagem.com.br/legislacaoread.asp?id=118. Acessado em: 06 dez. 2020.

8. CAFURE VA, GRACIOLLI-PATRIARCHA SR. Os resíduos de serviços de saúde e seus impactos ambiental; uma revisão bibliográfica. Interações, 2015; 16(2): 302-314.

9. CORRÊA LB, et al. O processo de formação em saúde: o saber resíduos sólidos de serviços de saúde em vivência práticas. Revista Brasileira de Enfermagem, 2007; 60 (1): 21-25.

10. DOI KM, MOURA MSS. Resíduos sólidos de serviços de saúde: uma fotografia do comprometimento da equipe de enfermagem. Revista Gaúcha de Enfermagem, 2011; 338-344.

11. GARBIN AJI, et al. A visão dos acadêmicos de odontologia sobre o gerenciamento dos resíduos do serviço de saúde. Archives of Health Investigation, 2015; 4(4): 63-67.

12. MARTINS DF, BENITO LAO. Florence Nightingale e as suas contribuições para o controle das infecções hospitalares Universitas: Ciências da Saúde, 2016; 14 (2):153-166.

13. MINAYO MCS. O desafio do conhecimento: pesquisa qualitativa em saúde, 2015.

14. Moreira, AMM., Gunther, W. Solid waste management in primary healthcare centers: application of a facilitation tool. Rev. Latino-Am. Enfermagem. 2016; 24: e2768.

15. MORESCHI C, et al. A importância dos resíduos de serviços de saúde para docentes, discentes e egressos da área da saúde. Revista Gaúcha de Enfermagem, 2014; 35(2): 20-26.

16. OLIVEIRA ACR, et al. Gerenciamento de Resíduos em laboratórios de uma universidade pública brasileira: um desafio para a saúde ambiental e a saúde do trabalhador, Saúde debate, 2020; 43 (Spe3). 
17. PEREIRA MS, et al. Gerenciamento de resíduos em unidades não hospitalares de urgência e emergência. Revista Latino-Americana de Enfermagem, 2013; 21 (espec.): t1-t8.

18. PETRES AA, DA ROS MA. A determinação social da saúde e a promoção da saúde. Arquivos Catarinenses de Medicina, 2018; 47 (3): 183-196.

19. SANCHEZ APM, et al. Resíduos de Serviços de Saúde: conhecimento de enfermeiros da Atenção Básica. Revista Brasileira de Enfermagem, 2018; 71 (5): 2508-2517.

20. SILVA DF, et al. Avaliação do gerenciamento dos resíduos de serviços de saúde em municípios da região metropolitana de Belo Horizonte (Brasil). Engenharia Sanitária e Ambiental, 2014; 19 (3): 251-262.

21. SILVA DP, et al. Percepção clínica, ética e legal de acadêmicos de Odontologia sobre gerenciamento de resíduos de serviços de saúde. Revista da ABENO, 2019; 19 (3):113-122.

22. SOARES MI, et al. A técnica de grupo focal na coleta de dados qualitativos: relato de experiência. REME - Revista Mineira de Enfermagem, 2016.

23. SZCZERBOWSKI AC, MORAIS CR. Manejo de resíduos sólidos em unidade básica de saúde da cidade de Estrela do Sul, Minas Gerais, Brasil. Getec, 2017; 6(11): 29-40.

24. VITAL MSB, GUIMARAES PSS. Plano de Gerenciamento de Resíduos de Serviços de Saúde: Descortinando o conhecimento dos Enfermeiros. GEP NEWS, 2018; 1 (1):167-171.

25. ZAJAC MAL, et al. Gerenciamento de resíduos de serviços de saúde (RSS) em um Hospital público: experiência de intervenção por parte de uma Universidade. Revista JHMREVIEW, 2016; 2 (2):44-62.

26. ZARPELÃO RZN, et al. Waste management in a petrochemical industry: a sustainable practice. International Journal of Engineering and Technical Research (IJETR), 2016; 32-36. 